\title{
Grape seed proanthocyanidin extract attenuates varicocele-induced testicular oxidative injury in rats by activating the Nrf2-antioxidant system
}

\author{
YONG WANG $^{1 *}$, FAN CHEN $^{1 *}$, MING LIANG ${ }^{1,2}$, SHOUZHEN CHEN $^{1}$, \\ YAOFENG ZHU ${ }^{1}$, ZHICHUAN ZOU ${ }^{1}$ and BENKANG SHI ${ }^{1}$ \\ ${ }^{1}$ Department of Urology, Qilu Hospital of Shandong University; ${ }^{2}$ Reproductive Medical Center, \\ The Second Hospital Affiliated to Shandong University of Traditional Chinese Medicine, \\ Jinan, Shandong 250000, P.R. China
}

Received December 27, 2015; Accepted February 1, 2017

DOI: $10.3892 / \mathrm{mmr} .2017 .8020$

\begin{abstract}
The present study investigated whether grape seed proanthocyanidin extract (GSPE) can attenuate varicocele-induced testicular oxidative injury through the nuclear factor (erythroid-derived 2)-like 2 (Nrf2) antioxidant pathway. A varicocele model in rats was established by partial ligation of the left renal vein. Following 4 weeks of GSPE administration, the decreased sperm count and motility and other pathological changes caused by varicocele were significantly alleviated, as indicated by the results of computer-assisted sperm analysis and hematoxylin and eosin staining. In addition, the decreased antioxidant enzyme (superoxide dismutase and glutathione peroxidase) activity and elevated oxidative stress level were partially reversed by administration of GSPE. Furthermore, the apoptotic level of the testis induced by varicocele was decreased by the GSPE treatment, according to terminal deoxynucleotidyl transferase dUTP nick end labeling assay. Additionally, the expression of apoptosis-related proteins, including B-cell lymphoma 2 (Bcl-2), Bcl-2-like protein 4 and cleaved caspase-3, were also affected by GSPE. GSPE activated Nrf2, which is a key antioxidative transcription factor, with elevation of the downstream factor hemeoxygenase-1. These findings suggest that GSPE can ameliorate abnormal spermatogenesis and testicular injury in varicocele rats, potentially due to its antioxidative activity and ability to activate the Nrf2 pathway.
\end{abstract}

Correspondence to: Professor Benkang Shi, Department of Urology, Qilu Hospital of Shandong University, 107 Wenhua Xi Road, Jinan, Shandong 250000, P.R. China

E-mail: benkangshi@126.com

${ }^{*}$ Contributed equally

Key words: grape seed proanthocyanidin extract, varicocele, oxidative stress, Nrf2, testis

\section{Introduction}

Varicocele, which is a dilation of the veins of the pampiniform plexus draining the testes, is present in $\sim 15 \%$ of all males and $19-41 \%$ of males diagnosed with primary infertility (1). The pathophysiological mechanism of varicocele remains to be elucidated but is possibly caused by blood flow occlusion in the spermatic vein, or hypofunction of its valves, causing blood stasis and making the spermatic vein coil and expand (2).

Previous studies $(3,4)$ have indicated that, in addition to decreasing sperm count and motility and causing DNA damage, varicocele may also disturb the development of the testes. Although the exact mechanism by which varicocele causes abnormal spermatogenesis remains to be elucidated, oxidative stress is regarded as one of the primary etiologic causes (5-7). Oxidative stress has been reported to affect the activity of the spermatozoa, to injure DNA structure and to accelerate apoptosis, all of which would lead to decreased sperm numbers and motility, abnormal development of morphology and impaired function of sperm in the testes (8). Enzymatic antioxidants in the testes, including glutathione peroxidase (GSH-Px), heme oxygenase-1 (HO-1) and NAD(P) H:quinone oxido-reductase-1, can scavenge free radicals to tackle oxidative stress $(9,10)$. Hence, upregulation of these enzymatic antioxidants can prevent the damage to the testis and spermatozoa caused by oxidative stress (11).

Nuclear factor E2-related factor 2 (Nrf2) coordinately modulates the expression of $>200$ genes in humans and other animals, acting as an endogenous antioxidant mechanism (12). The Nrf2-antioxidant-response element (ARE) signaling pathway is hypothesized to act as one of the major defense systems, which may inhibit oxidative stress via promoting the expression of HO-1 and other downstream antioxidative genes (13). Furthermore, Nrf2 combines with Kelch-like ECH-associated protein 1 (KEAP1) and remains in the cytoplasm without oxidative stimulation (14). KEAP1 can promote the ubiquitylation of $\mathrm{Nrf} 2$ to repress its function. However, when excess reactive oxygen species (ROS) are present, KEAP1 becomes inactivated (15) and Nrf2 translocates into 
the nucleus and binds to the ARE to initiate the transcription of downstream genes $(11,14)$.

Grape seed proanthocyanidin extract (GSPE) consists of a group of polyphenolic bioflavonoids that possess a broad spectrum of biological, pharmacological and therapeutic properties that combat oxidative stress (16). GSPE has been reported (17) to demonstrate more powerful antioxidative activity than vitamin $C$ or E. Previous studies (18-20) have demonstrated that GSPE can activate the Nrf2 defense pathway to exert its protective function in various situations, including diabetes mellitus, nickel sulfate poisoning and arsenic poisoning.

The present study aimed to investigate whether GSPE can alleviate varicocele-induced oxidative injury by activating the Nrf2 defense pathway in rat testes.

\section{Materials and methods}

Reagents. GSPE (lot.no. G050412) was purchased from Tianjin Jianfeng, Inc. (Tianjin, China). Rabbit anti-Nrf2 antibody (cat. no. ab137550), mouse anti-HO-1 antibody (cat. no. ab13243), rabbit anti-H2A histone family member X (H2A.X) antibody (cat. no. ab11175) and rabbit anti-B-cell lymphoma 2 (Bcl-2)-like protein 4 (Bax) antibody (cat. no. ab32503) were all purchased from Abcam (Cambridge, UK); rabbit anti-Bcl-2 antibody (cat. no. sc-783), mouse anti-actin antibody (cat. no. sc-58673), goat-anti-rabbit immunoglobulin G (IgG)-horseradish peroxidase (HRP) (cat. no. sc-2004) and goat-anti-mouse IgG-HRP (cat. no. sc-2005) were all purchased from Santa Cruz Biotechnology, Inc. (Dallas, TX, USA); rabbit anti-cleaved-caspase-3 (cat. no. 9661) was purchased from Cell Signaling Technology, Inc. (Danvers, MA, USA). The total superoxide dismutase (SOD) assay, GSH-Px assay andmalondialdehyde (MDA) assay kits were obtained from Nanjing Jiancheng Bioengineering Institute (Nanjing, China). The nuclear and cytoplasmic protein extraction, bicinchoninic acid (BCA) assay and terminal deoxynucleotidyl transferase dUTP nick end labeling (TUNEL) apoptosis assay kits were all purchased from Beyotime Institute of Biotechnology (Beijing, China).

Animals and groups. Adult (6-8 weeks old) male Wistar rats weighing 280-320 g were supplied by the Animal Center of Shandong University (license no. SCXK20130007). The animals were supplied with food and water and were maintained on a standard $12 \mathrm{~h}$ light:dark cycle with $60 \%$ relative humidity and a temperature of $25^{\circ} \mathrm{C}$. Animal care and management was approved by the Ethics Committee of Qilu Hospital of Shandong University (approval no. DWLL-20B-025; Jinan, China). Anesthesia was induced by an intraperitoneal injection of $10 \%$ chloral hydrate and animals were sacrificed using cervical dislocation.

The rats were randomly divided into six groups (10 rats/group) following 7 days of acclimatization: i) Control; ii) control + GSPE; iii) sham; iv) sham + GSPE; v) varicocele (V); and vi) varicocele + GSPE.

Establishment of varicocele model. The procedure for establishing a varicocele model was previously reported by Tuner (21). The rats in the varicocele and varicocele + GSPE groups were anesthetized by intraperitoneal injection of $10 \%$ chloral hydrate $(3 \mathrm{ml} / \mathrm{kg})$. A midline laparotomy incision was made and the abdominal contents were gently pushed to the right side to expose the left renal vein, inferior vena cava and left spermatic vein. The adipose tissue surrounding the left renal vein was carefully dissected and a 4/0 silk suture was loosely placed around it, just distal to the insertion of the left spermatic vein. A rigid guide wire $(0.8 \mathrm{~mm}$ in diameter) was placed over the left renal vein and the suture was tied around the vein over it. The guide wire was then carefully removed. Congestion of the left renal vein and spermatic vein in each animal was immediately apparent. The incision was closed in two layers. The rats in the sham and sham + GSPE groups underwent the same procedure, however the sutures were not tied down. The rats were fasted for $24 \mathrm{~h}$ prior to and following surgery. The varicocele model was considered to be successfully established when the diameter of the left spermatic vein reached more than twice its previous size.

GSPE administration. At 4 weeks after surgery, the rats in the control + GSPE, sham + GSPE and varicocele + GSPE groups received an intragastric dose of $250 \mathrm{mg} / \mathrm{kg}$ GSPE daily for another 4 weeks, while the rats in the other three groups received the same volume of normal saline, also intragastrically.

Histological analysis. All the animals were euthanized following 4 weeks of GSPE or normal saline administration. The left testis was removed using a low abdominal incision. Half of the testis was put into liquid nitrogen and then stored at $-80^{\circ} \mathrm{C}$. The other half was fixed in $4 \%$ paraformaldehyde, dehydrated sequentially with ethanol and embedded in paraffin wax. The wax-embedded tissue was sliced into $5 \mu \mathrm{m}$ thick sections. Five sections per rat were stained with hematoxylin and eosin (H\&E) for morphological analysis.

Immunohistochemistry of Nrf2. The sections were placed in $0.01 \mathrm{M}$ sodium citrate buffer and incubated between $92-98^{\circ} \mathrm{C}$ to perform antigen retrieval. The sections were then incubated in $3 \% \mathrm{H}_{2} \mathrm{O}_{2}$ for $30 \mathrm{~min}$ and then blocked with $10 \%$ normal goat serum (OriGene Technologies, Inc., Rockville, MD, USA) for $30 \mathrm{~min}$ at $37^{\circ} \mathrm{C}$. The sections were then incubated with rabbit polyclonal antibody specific for Nrf2 (dilution 1:200) overnight at $4^{\circ} \mathrm{C}$. The sections were then incubated with goat polyclonal secondary antibody (1:500) to rabbit for $30 \mathrm{~min}$. Finally, the sections were stained using a 3,3'-diaminobenzidine kit and then stained with hematoxylin and observed with a light microscope.

Sperm counts and motility. The left cauda epididymis tissue was chopped into small pieces and incubated in $3 \mathrm{ml}$ normal saline in a $37^{\circ} \mathrm{C}$ water bath for $2 \mathrm{~min}$. Approximately $10 \mu \mathrm{l}$ of sperm suspension was dropped onto a blood cell counting plate. The sperm concentration and rate of sperm motility (progressive + non-progressive) were measured by computer-assisted sperm analysis (Microptic, Barcelona, Spain).

Western blotting. As described above, half of the testis was stored at $-80^{\circ} \mathrm{C}$. The nuclear and cytoplasmic proteins of the testis were extracted according to the protocol of the nuclear 
A

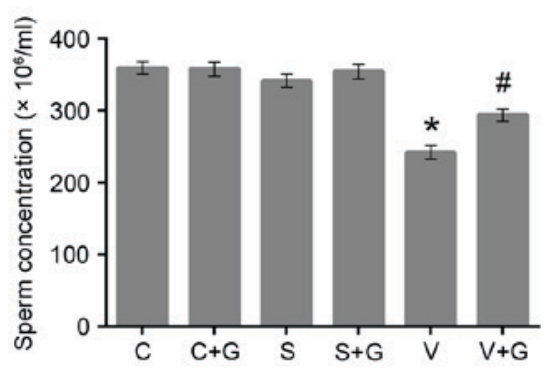

B

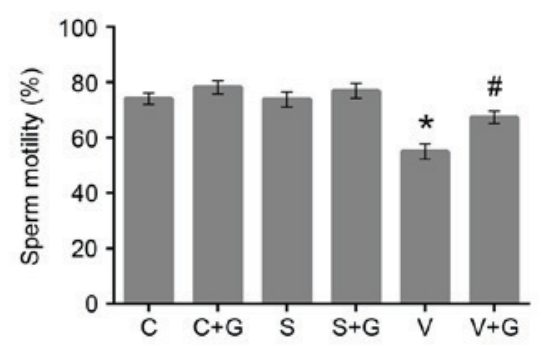

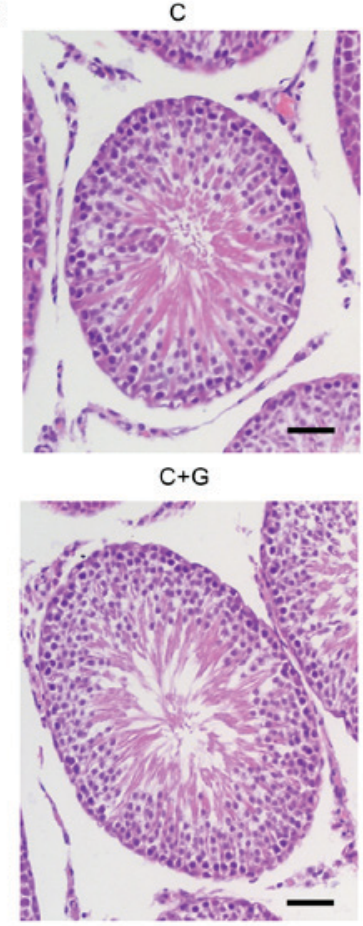

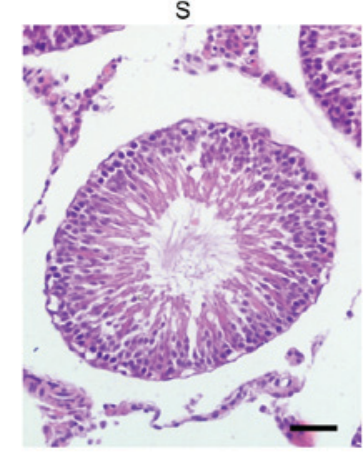

$S+G$

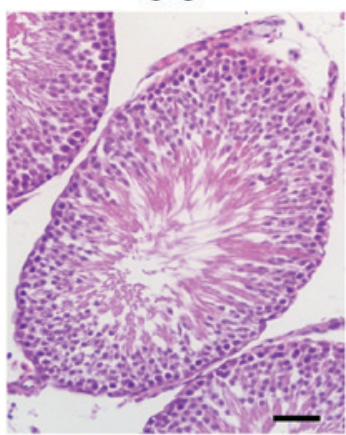

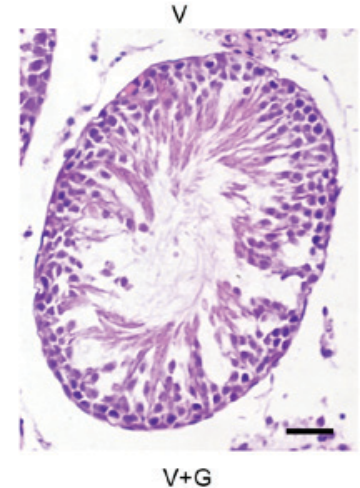

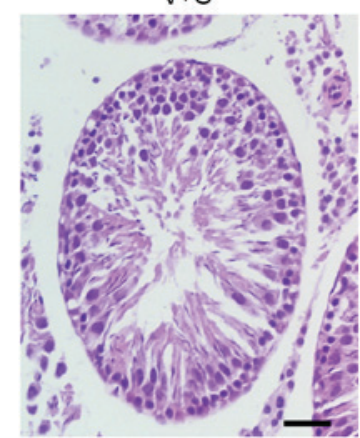

Figure 1. Morphological and functional evaluations of the testis. (A) Statistical results of testicular sperm concentration. ${ }^{*} \mathrm{n}=9$, $\mathrm{P}=0.000 \mathrm{vs}$. control group; ${ }^{\#} \mathrm{n}=9, \mathrm{P}=0.013$ vs. varicocele group. (B) Statistical results of testicular sperm motility. ${ }^{*} \mathrm{n}=9, \mathrm{P}=0.001$ vs. control group; ${ }^{*} \mathrm{n}=9$, $\mathrm{P}=0.033 \mathrm{vs}$. varicocele group. (C) Histological sections of the left testis with hematoxylin and eosin staining exhibit fewer and looser testicular epithelial cells with structural damage in the varicocele group compared with the control group, whereas GSPE can constrain the varicocele-triggered morphological changes. Scale bar, $50 \mu \mathrm{m}$. C, control group; C+G, control + GSPE group; S, sham group; S+G, sham + GSPE group; V, varicocele group; V+G, varicocele + GSPE group; GSPE, grape seed proanthocyanidin extract.

and cytoplasmic protein extraction kit (cat. no. P0028; Beyotime Institute of Biotechnology). The concentration of the proteins was measured by the BCA method. Then proteins $(50 \mu \mathrm{g})$ were fractionated by $10 \%$ SDS-PAGE and transferred onto PVDF membranes. The membranes were blocked in 5\% milk for $2 \mathrm{~h}$ and then incubated with primary antibodies specific for Nrf2 (1:500), HO-1 (1:500), H2A.X (1:500), $\beta$-actin (1:1,000), cleaved caspase-3 (1:500), Bcl-2 $(1: 1,000)$ or $\operatorname{Bax}(1: 1,000)$ at $4^{\circ} \mathrm{C}$ overnight. Then the membrane was incubated with secondary antibodies $(1: 5,000)$ at room temperature for $2 \mathrm{~h}$ and visualized using ECL reagent (Beyotime Institute of Biotechnology). The intensity of the blotted bands was analyzed by Quantity One software (version 4.2; Bio-Rad Laboratories, Inc., Hercules, CA, USA). H2A.X and $\beta$-actin were used as loading controls for nuclear and total protein, respectively.

Measurement of oxidative stress. Oxidative stress in the testes was evaluated by assessing the concentration of MDA, SOD and GSH-Px. The concentrations of MDA, SOD and GSH-Px were tested according to the protocols of the lipid peroxidation MDA assay, total SOD assay kit with WST-8 and cellular GSH-Px assay kits (Beyotime Institute of Biotechnology).

TUNEL assay. TUNEL assays were conducted to evaluate the apoptotic level of the testes using the One-Step TUNEL apoptosis assay kit (Beyotime Institute of Biotechnology). After being incubated with the TUNEL reagent in humid and dark conditions for $60 \mathrm{~min}$, the sections were stained with DAPI (Sigma-Aldrich; Merck KGaA, Darmstadt, Germany) for $10 \mathrm{~min}$ and observed under a fluorescence microscope. The apoptotic cells exhibited red fluorescence when excited by light of $550 \mathrm{~nm}$, while the nucleus exhibited blue fluorescence when excited by light of $358 \mathrm{~nm}$. TUNEL-positive cells were counted per $10^{3}$ cells.

Statistical analysis. All the measured data were presented as the mean \pm standard deviation ( $n \geq 5$ in each group). One-way 
analysis of variance was used to make comparisons between different groups followed by Dunnett's test to perform comparisons between two groups. $\mathrm{P}<0.05$ was considered to indicate a statistically significant difference. All of the data were analyzed using SPSS version 19.0 (IBM SPSS, Armonk, NY, USA).

\section{Results}

Effects of GSPE on morphology and function of testes. As demonstrated in Fig. 1A and B, sperm concentration and motility (progressive + nonprogressive) tended to be significantly lower in the varicocele compared with the control group ( $\mathrm{P}=0.000$ and $\mathrm{P}=0.001$, respectively). However, compared with the varicocele group, GSPE ameliorated the sperm concentration and motility in the varicocele + GSPE group $(\mathrm{P}=0.013$ and $\mathrm{P}=0.033$, respectively).

H\&E staining demonstrated that varicocele caused damaging effects to the compared with the control and sham groups (Fig. 1C). The number testicular epithelial cells in the varicocele group were observably fewer and were looser than those in the control and sham groups. Abnormal spermatogenic cell types in damaged seminiferous tubules were also observed in the testicular sections of the varicocele group. However, the varicocele + GSPE group exhibited limited histological changes, which indicated that GSPE may alleviate the damage triggered by varicocele.

GSPE attenuated the oxidative stress in varicocele testes. SOD, GSH-Px and MDA activity were measured to evaluate the oxidative stress occurring in the left rat testis. The concentrations of SOD and GSH-Px were significantly lower in the varicocele group compared with the control or sham groups $(\mathrm{P}<0.05)$. By contrast, administration of GSPE markedly restored SOD and GSH-Px concentrations $(\mathrm{P}=0.018$ and $\mathrm{P}=0.020$, respectively; Fig. $2 \mathrm{~B}$ and $\mathrm{C})$. Compared with the control and sham groups, the level of MDA was much higher due to the effect of varicocele $(\mathrm{P}=0.000)$. GSPE can decrease the level of MDA in the testes of varicocele rats significantly ( $\mathrm{P}=0.007$; Fig. $2 \mathrm{~A})$. In addition, according to the results of the control + GSPE and sham + GSPE groups, no evident effects of GSPE were observed on the levels of MDA, SOD or GSH-Px $(\mathrm{P}>0.05)$ in the control or sham groups.

Protective effects of GSPE on apoptosis in varicocele testis. Several studies $(8,22)$ have demonstrated that varicocele can increase the apoptosis level in the testis due to oxidative stress. The present study examined whether GSPE had any protective effects on the apoptosis level of varicocele testes by TUNEL staining. The number of TUNEL-positive cells in the varicocele group was markedly increased compared with the control group ( $\mathrm{P}=0.004)$, whereas GSPE significantly decreased TUNEL-positive cell numbers in the varicocele rats $(\mathrm{P}=0.026$; Fig. 3A and B). In addition, western blotting demonstrated that the expression of cleaved caspase- 3 in the testis of the varicocele group increased significantly compared with the control group $(\mathrm{P}=0.006)$, whereas treatment of varicocele with GSPE decreased the expression of cleaved caspase-3 significantly ( $\mathrm{P}=0.028$; Fig. 3C).
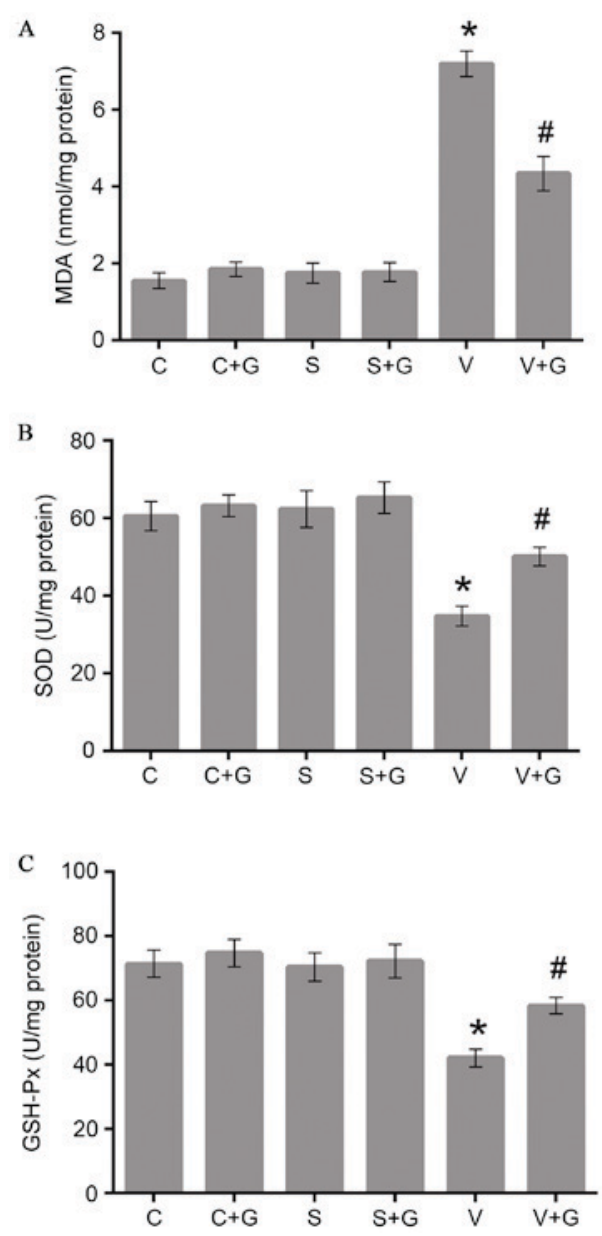

Figure 2. Effect of varicocele and GSPE on oxidative stress in the testis (A) MDA level in the testis. ${ }^{*} \mathrm{n}=6, \mathrm{P}=0.000$ vs. control group; ${ }^{*} \mathrm{n}=6, \mathrm{P}=0.007$ vs. varicocele group. (B) SOD activity in the testis. ${ }^{*} \mathrm{n}=6, \mathrm{P}=0.004$ vs. control group; ${ }^{\#} \mathrm{n}=6, \mathrm{P}=0.018$ vs. varicocele group. (C) GSH-Px activity in the testis. ${ }^{*} \mathrm{n}=6, \mathrm{P}=0.004$ vs. control group; ${ }^{n} \mathrm{n}=6, \mathrm{P}=0.020$ vs. varicocele group. GSPE, grape seed proanthocyanidin extract; MDA, malondialdehyde; $\mathrm{C}$, control group; $\mathrm{C}+\mathrm{G}$, control + GSPE group; $\mathrm{S}$, sham group; $\mathrm{S}+\mathrm{G}$, sham + GSPE group; V, varicocele group; $\mathrm{V}+\mathrm{G}$, varicocele + GSPE group; SOD, superoxide dismutase; GSH-Px, glutathione peroxidase.

The mitochondrial apoptotic pathway has been reported (23) to be involved in varicocele testes, therefore whether GSPE can avert the activation of the mitochondrial apoptotic pathway in varicocele testes was investigated. Western blotting demonstrated that the Bax to Bcl-2 expression ratio was significantly higher in the varicocele group compared with the control group $(\mathrm{P}=0.000)$. GSPE significantly alleviated the increase of the Bax to Bcl-2 expression ratio in the testes of the varicocele rats $(\mathrm{P}=0.015$; Fig. 3D).

GSPE exerted protective effects through activation of the Nrf2 pathway. The transcription factor Nrf2 has an important role in regulating the cellular antioxidant system. The effect of GSPE on the expression of Nrf2 was investigated and immunohistochemical results demonstrated that varicocele can slightly increase the expression of Nrf2 in interstitial cells compared with the control group, while administration of GSPE markedly increased the expression of Nrf2 in interstitial cells in the three groups that were administered GSPE (Fig. 4A). The expression of Nrf2 was predominantly located in the nucleus 
A
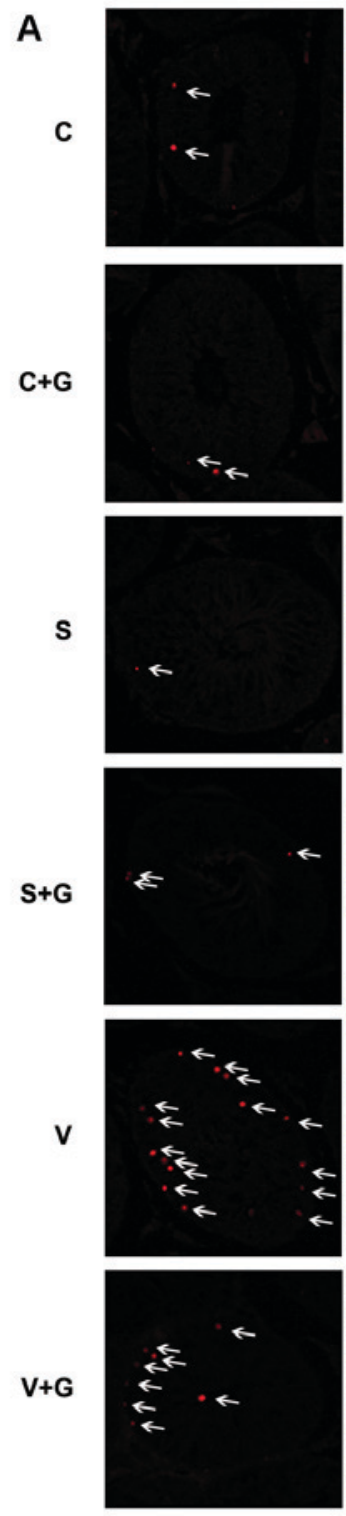
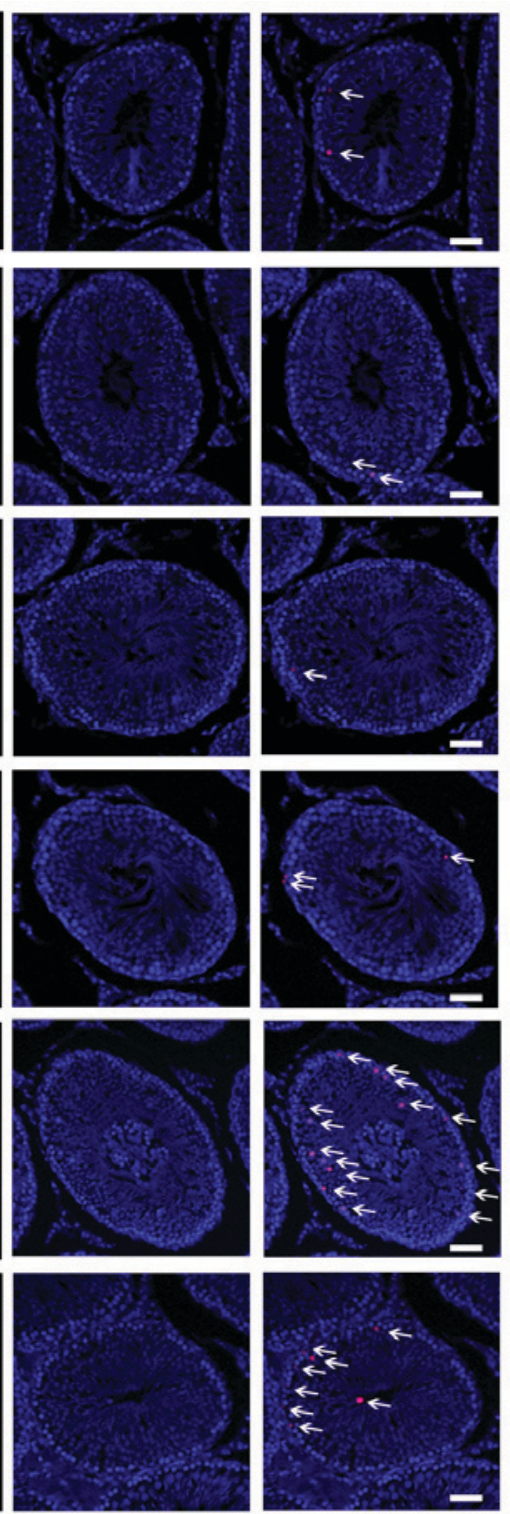
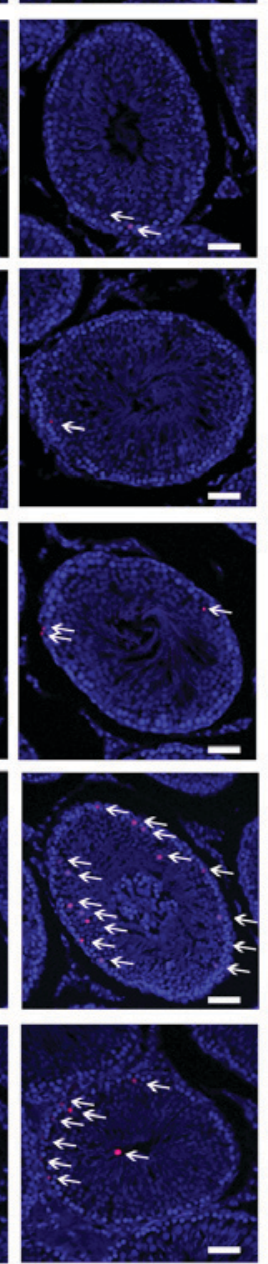

B

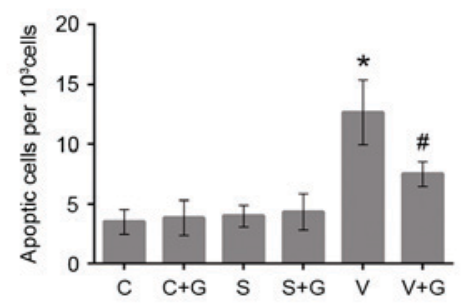

C
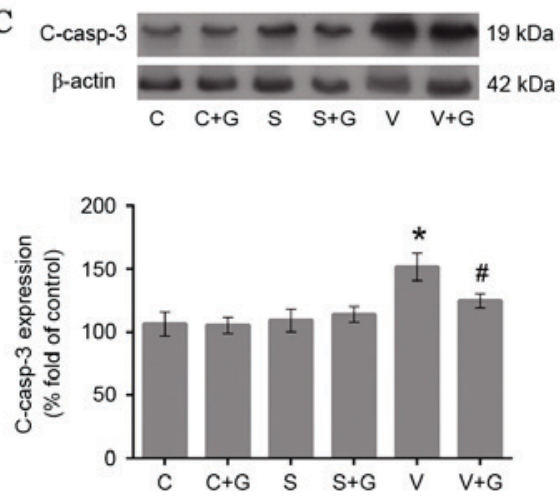

D
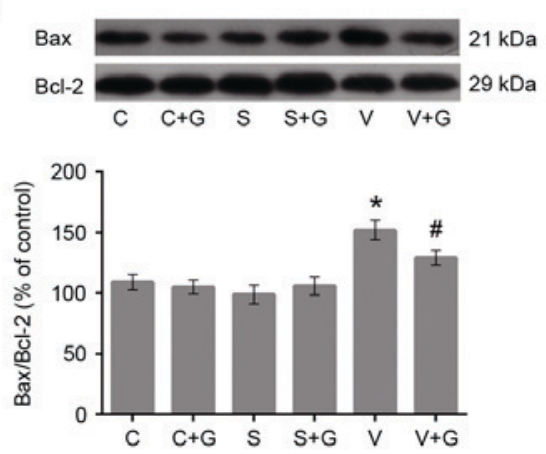

Figure 3. Effect of GSPE on apoptosis in the testis. (A) TUNEL staining showing cell apoptosis in the testis. The arrows indicate TUNEL-positive cells (red), the nuclei stained with DAPI (blue). Scale bar, $50 \mu \mathrm{m}$. (B) Statistical results of TUNEL staining, " $\mathrm{n}=6, \mathrm{P}=0.004$ vs. control group; " $\mathrm{n}=6$, $\mathrm{P}=0.026 \mathrm{vs}$. varicocele group. (C) The expression of c-casp-3 protein in the testis. " $\mathrm{n}=5, \mathrm{P}=0.006$ vs. the control group; ${ }^{*} \mathrm{n}=5, \mathrm{P}=0.028$ vs. the varicocele group. (D) The Bax to $\mathrm{Bcl}-2$ protein expression ratio in the testis. ${ }^{n} \mathrm{n}=5, \mathrm{P}=0.000$ vs. control group; ${ }^{\prime} \mathrm{n}=5, \mathrm{P}=0.015$ vs. varicocele group. GSPE, grape seed proanthocyanidin extract; TUNEL, terminal deoxynucleotidyl transferase dUTP nick end labeling; C, control group; C+G, control + GSPE group; S, sham group; S+G, sham + GSPE group; V, varicocele group; V+G, varicocele + GSPE group; Bax, Bcl-2-like protein 4; Bcl-2, B-cell lymphoma 2; c-casp-3, cleaved caspase-3.

of the interstitial cells. Western blotting demonstrated that the varicocele group expressed a significantly higher level of Nrf2 than the control group ( $\mathrm{P}=0.044$; Fig. 4B), and the varicocele + GSPE group demonstrated a significantly higher level of Nrf2 expression compared with the varicocele group ( $\mathrm{P}=0.006$; Fig. 4B). The groups administered with GSPE all demonstrated significantly higher expression of Nrf2 compared with the control group $(\mathrm{P}<0.01$ in three groups; Fig. 4B).

Previous studies $(24,25)$ have demonstrated that Nrf2, as a transcription factor, can only exert its function by entering the nucleus. Therefore, the nuclear expression of Nrf2 was measured. As demonstrated in Fig. 4C, the varicocele group expressed a higher level of Nrf2 than the control group $(\mathrm{P}=0.014)$. The varicocele + GSPE group expressed a significantly increased level of Nrf2 than the varicocele group $(\mathrm{P}=0.034)$. The groups treated with GSPE all demonstrated a higher level of nuclear Nrf2 expression than the control group $(\mathrm{P}<0.01)$.

The expression of HO-1, a gene downstream of Nrf2, was next measured to evaluate the antioxidative effect of Nrf2. As demonstrated in Fig. 4D, the varicocele + GSPE group demonstrated higher expression of HO-1 than the varicocele group $(\mathrm{P}=0.022)$. However, no significant differences were demonstrated when comparing the expression of total $\mathrm{Nrf} 2$, nuclear Nrf2 or HO-1 between the three groups that were given GSPE (Fig. 4).

\section{Discussion}

Varicocele is an abnormal tortuosity of the pampiniform plexus of the spermatic cord, which has incidence rates of $4.4-22.6 \%$ in the male population, particularly in males with 
A
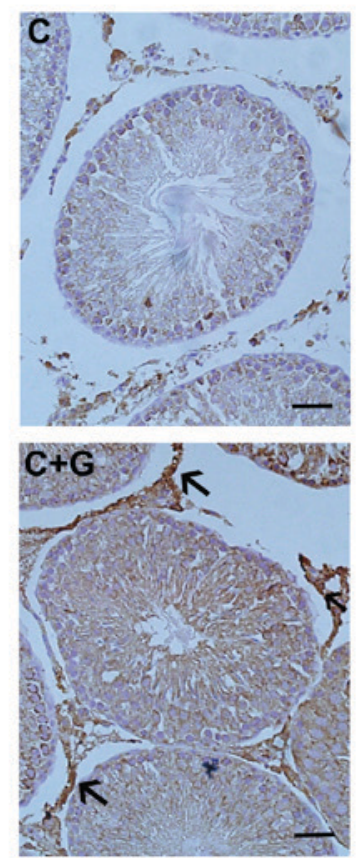
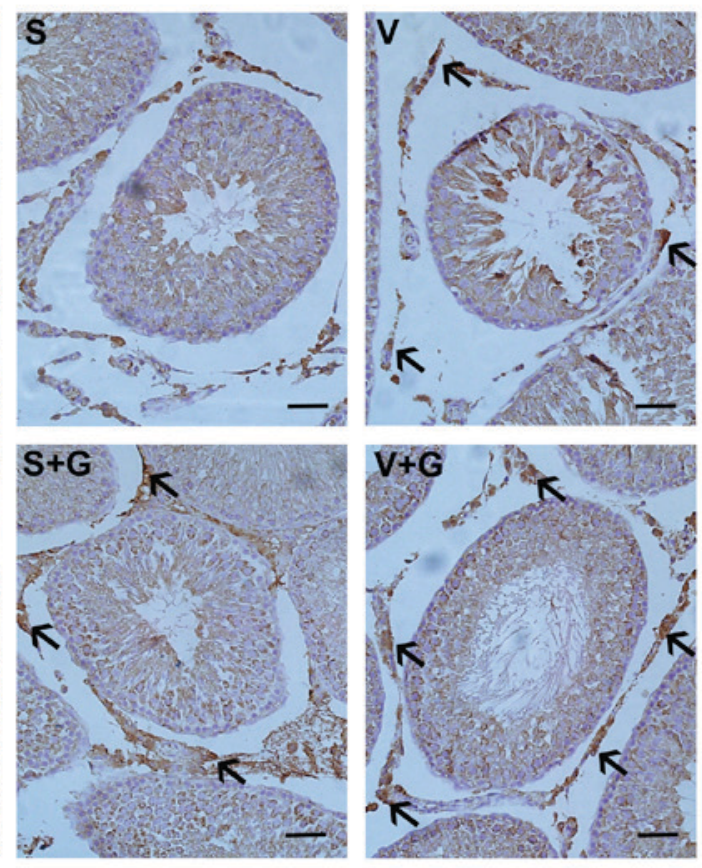

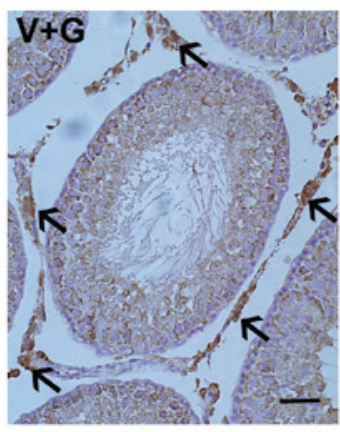

B

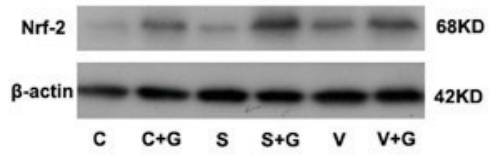

C

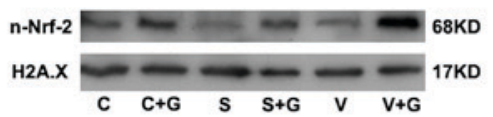

D

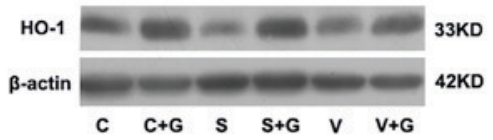

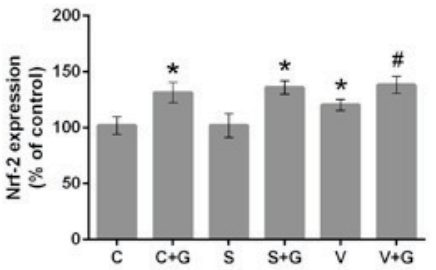


Figure 4. Effect of GSPE on the Nrf2 pathway in the testis. (A) Immunohistochemical staining results for Nrf2 in the testis. The arrow shows Nrf2 positive staining predominantly located in the nucleus of the interstitial cells. Scale bar: $50 \mu \mathrm{m}$. (B) The protein expression of total Nrf2 in the testis ${ }^{*} \mathrm{n}=5, \mathrm{C}+\mathrm{G}(\mathrm{P}=0.004), \mathrm{S}+\mathrm{G}(\mathrm{P}=0.002), \mathrm{V}(\mathrm{P}=0.044)$ vs. control group; ${ }^{*} \mathrm{n}=5, \mathrm{P}=0.006$ vs. varicocele group. (C) The protein expression of $\mathrm{n}-\mathrm{Nrf} 2$ in the testis. " $\mathrm{n}=5, \mathrm{C}+\mathrm{G}(\mathrm{P}=0.0024), \mathrm{S}+\mathrm{G}(\mathrm{P}<0.001), \mathrm{V}(\mathrm{P}=0.014)$ vs. control group; ${ }^{\#} \mathrm{n}=5, \mathrm{P}=0.034$ vs. varicocele group. (D) The protein expression of HO-1 in the testis. ${ }^{*} \mathrm{n}=5, \mathrm{C}+\mathrm{G}(\mathrm{P}<0.001), \mathrm{S}+\mathrm{G}(\mathrm{P}<0.001), \mathrm{V}(\mathrm{P}=0.039)$ vs. control group; ${ }^{*} \mathrm{n}=5, \mathrm{P}=0.022$ vs. varicocele group. GSPE, grape seed proanthocyanidin extract; C, control group; $\mathrm{C}+\mathrm{G}$, control + GSPE group; S, sham group; $\mathrm{S}+\mathrm{G}$, sham + GSPE group; V, varicocele group; V+G, varicocele + GSPE group; n, nuclear; Nrf2, nuclear factor (erythroid-derived 2)-like 2; H2A.X, H2A histone family member X; HO-1, heme oxygenase-1.

primary or secondary infertility. Many associated factors are considered relevant in the pathophysiology of varicocele. By using a well-established rat model of varicocele (21), the injured spermatogenic function and pathologic alterations of the testes in the varicocele group was demonstrated. In addition, the sperm concentration and motility (based on computer assisted semen analysis) and the histological results of $\mathrm{H} \& \mathrm{E}$ staining were clearly ameliorated following 4 weeks of GSPE administration, which illustrated that GSPE can attenuate the functional and morphologic damage to rat testes and epididymis caused by varicocele.

GSPE, chemically a mixture of pycnogenol and flavonoid including oligomeric proanthocyanidins, is a potent antioxidant (19). The biologically active compounds possess protective effects against oxidative stress induced by free radicals and ROS. Studies $(19,26,27)$ have demonstrated that 
the administration of GSPE can ameliorate oxidative damage from various causes. First, in accordance with previous findings (28), the present study demonstrated that varicocele can result in deterioration of oxidative stress markers. Additionally, in the varicocele + GSPE group, the testicular activity of GSH-Px and SOD were higher than those in the varicocele group. MDA concentrations were lower in the varicocele + GSPE group. These results proved that GSPE can partially relieve the oxidative stress in testes induced by varicocele.

Apoptosis is one of the dominant mechanisms of testicular dysfunction resulting from varicocele (29-32). Varicocele has been reported (33-35) to increase sperm DNA damage and pachytene spermatocyte programmed death. The TUNEL assay results also identified an increased apoptosis level in the spermatogonia of the varicocele group compared with the control group. This finding was compatible with the protein expression of Bcl-2, Bax and cleaved caspase-3 (Fig. 3C and D). Conversely, GSPE has been demonstrated $(20,36,37)$ to protect cells from the apoptosis induced by oxidative stress. The number of apoptotic nuclei per $10^{3}$ cells revealed less cell DNA damage in the varicocele + GSPE group compared with the varicocele group. Furthermore, the ratio of Bax to Bcl-2 and activated caspase- 3 expression fell in the varicocele + GSPE group. Caspase-3 is considered to be a major executioner protease and the ratio of $\mathrm{Bax}$ to $\mathrm{Bcl}-2$ is also a good indicator of apoptosis levels (38). Consequently, it was hypothesized that the administration of GSPE for 4 weeks was able to alleviate the apoptosis in the testicular tissues of rats with varicocele.

However, the exact mechanism of the elevated oxidative stress and apoptosis in male reproductive organs has yet to be fully elucidated. Nrf2 is a transcription factor that improves the expression of antioxidant proteins and can function against oxidative damage (39). Nrf2 is known to be associated with the testicular apoptosis induced by diabetes (40). Similarly, the results of the present study revealed higher expression of Nrf2 and its downstream gene HO-1 in the varicocele group compared with the control group due to the oxidative stimulation caused by varicocele (Fig. 4). Several studies $(18,41)$ have demonstrated that GSPE can activate the Nrf2 pathway to exert its antioxidative function. However, few reports have focused on the correlation between GSPE and the Nrf2 signaling pathway in the testis. The present study revealed that Nrf2 and HO-1 were significantly upregulated due to the administration of GSPE compared to the varicocele and control groups. In addition, the immunohistochemical staining results for $\mathrm{Nrf} 2$ were in agreement with the western blot analysis. Thus, GSPE may exert a sustained effect on Nrf2 expression, which can further attenuate the oxidative stress and apoptosis triggered by varicocele in rat testes. However, the results demonstrated that the control + GSPE, sham + GSPE and varicocele + GSPE groups all had significantly higher expression of Nrf2 and its downstream gene HO-1, which suggests that GSPE activates the Nrf2 pathway in normal and varicocele rat testis.

Admittedly, there may also be several limitations in the present study. The rat model of varicocele cannot be used to interpret the equivalent situation in human patients due to the anatomical structure differences of the left spermatic vein between rats and humans and different standards for semen analysis. In addition, the present study did not adopt different concentration gradients or time courses of GSPE treatment. It is possible that employing a different dose or period of gavage would reinforce the functions of GSPE. Future studies may compare the different effects of GSPE with internal spermatic vein ligation, which is the main surgical treatment for varicocele in clinics (42).

In summary, the data from the present study indicated that varicocele leads to oxidative and mitochondrial apoptotic injuryand GSPE upreg ulates Nrf2 and its downstream genes to mitigate such damage and resume normal spermatogenic organ function. Thus, GSPE appears to be an effective protective agent that may be used in the treatment of male varicocele in the future.

GSPE, which consists of a group of polyphenolic bioflavonoids, possesses potent antioxidative activity. Oxidative stress is one of the major pathogenic mechanisms in varicocele, and can cause morphological and functional damage to the testis and epididymis. The present study confirmed that GSPE can activate the Nrf2-antioxidant system to attenuate varicocele induced testicular injury caused by oxidative stress.

\section{Acknowledgements}

This work was supported by the National Natural Science Foundation of China (grant nos. 81470987 and 81670687 to B. Shi), the Tai Shan Scholar Foundation to B. Shi and the Natural Science Foundation of Shandong Province (grant no. ZR2014HQ062 to Y. Zhu). The authors thank Dr Yongxin Zou for providing associated experimental equipment and reagents.

\section{References}

1. Pastuszak AW and Wang R: Varicocele and testicular function. Asian J Androl 17: 659-667, 2015.

2. Liang M, Wen J, Dong Q, Zhao LG and Shi BK: Testicular hypofunction caused by activating p53 expression induced by reactive oxygen species in varicocele rats. Andrologia 47: 1175-1182, 2015.

3. Razi M and Malekinejad H: Varicocele-induced infertility in animal models. Int J Fertil Steril 9: 141-149, 2015.

4. Shiraishi K, Takihara $\mathrm{H}$ and Matsuyama $\mathrm{H}$ : Effects of grade 1 varicocele detected in the pediatric age-group on testicular development. J Pediatr Surg 44: 1995-1998, 2009.

5. Sohrabipour S, Jafari A, Kamalinejad M, Sarrafnejd A, Shahrestany T and Sadeghipour HR: The role of flaxseed and vitamin $\mathrm{E}$ on oxidative stress in prepubertal rats with experimental varicocele: An experimental study. Iran J Reprod Med 11: 459-466, 2013.

6. Razi M, Sadrkhanloo RA, Malekinejad H and Sarrafzadeh-Rezaei F: Testicular biohistochemical alterations following experimental varicocele in rats. Iran J Reprod Med 10: 209-218, 2012.

7. Altunoluk B, Efe E, Kurutas EB, Gul AB, Atalay F and Eren M: Elevation of both reactive oxygen species and antioxidant enzymes in vein tissue of infertile men with varicocele. Urol Int 88: 102-106, 2012.

8. Walczak-Jedrzejowska R, Wolski JK and Slowikowska-Hilczer J: The role of oxidative stress and antioxidants in male fertility. Cent European J Urol 66: 60-67, 2013.

9. Hayes JD and McLellan LI: Glutathione and glutathionedependent enzymes represent a co-ordinately regulated defence against oxidative stress. Free Radic Res 31: 273-300, 1999.

10. Hong CC, Ambrosone CB, Ahn J, Choi JY, McCullough ML, Stevens VL, Rodriguez C, Thun MJ and Calle EE: Genetic variability in iron-related oxidative stress pathways ( Nrf2, NQ01, NOS3, and HO-1), iron intake, and risk of postmenopausal breast cancer. Cancer Epidemiol Biomarkers Prev 16: 1784-1794, 2007.

11. Li Y, Cao Y, Wang F, Pu S, Zhang Y and Li C: Tert-butylhydroquinone attenuates scrotal heat-induced damage by regulating Nrf2-antioxidant system in the mouse testis. Gen Comp Endocrinol 208: 12-20, 2014. 
12. Hayes JD and McMahon M: NRF2 and KEAP1 mutations: Permanent activation of an adaptive response in cancer. Trends Biochem Sci 34: 176-188, 2009.

13. Wang R, Paul VJ and Luesch H: Seaweed extracts and unsaturated fatty acid constituents from the green alga Ulva lactuca as activators of the cytoprotective Nrf2-ARE pathway. Free Radic Biol Med 57: 141-153, 2013.

14. Kobayashi A, Kang MI, Okawa H, Ohtsuji M, Zenke Y, Chiba T, Igarashi $\mathrm{K}$ and Yamamoto $\mathrm{M}$ : Oxidative stress sensor Keap1 functions as an adaptor for Cul3-based E3 ligase to regulate proteasomal degradation of Nrf2. Mol Cell Biol 24: 7130-7139, 2004.

15. McMahon M, Lamont DJ, Beattie KA and Hayes JD: Keap1 perceives stress via three sensors for the endogenous signaling molecules nitric oxide, zinc, and alkenals. Proc Natl Acad Sci USA 107: 18838-18843, 2010.

16. Zhang Z, Zheng L, Zhao Z, Shi J, Wang X and Huang J: Grape seed proanthocyanidins inhibit $\mathrm{H}_{2} \mathrm{O}_{2}$-induced osteoblastic MC3T3-E1 cell apoptosis via ameliorating $\mathrm{H}_{2} \mathrm{O}_{2}$-induced mitochondrial dysfunction. J Toxicol Sci 39: 803-813, 2014.

17. Bagchi D, Garg A, Krohn RL, Bagchi M, Tran MX and Stohs SJ: Oxygen free radical scavenging abilities of vitamins $\mathrm{C}$ and $\mathrm{E}$, and a grape seed proanthocyanidin extract in vitro. Res Commun Mol Pathol Pharmacol 95: 179-189, 1997.

18. Chen S, Zhu Y, Liu Z, Gao Z, Li B, Zhang D, Zhang Z, Jiang X, Liu Z, Meng L, et al: Grape seed proanthocyanidin extract ameliorates diabetic bladder dysfunction via the activation of the Nrf2 pathway. PLoS One 10: e0126457, 2015.

19. Li SG, Ding YS, Niu Q, Xu SZ, Pang LJ, Ma RL, Jing MX, Feng GL, Liu JM and Guo SX: Grape seed proanthocyanidin extract alleviates arsenic-induced oxidative reproductive toxicity in male mice. Biomed Environ Sci 28: 272-280, 2015.

20. Su L, Deng Y, Zhang Y, Li C, Zhang R, Sun Y, Zhang K, Li J and Yao S: Protective effects of grape seed procyanidin extract against nickel sulfate-induced apoptosis and oxidative stress in rat testes. Toxicol Mech Methods 21: 487-494, 2011.

21. Turner TT: The study of varicocele through the use of animal models. Hum Reprod Update 7: 78-84, 2001.

22. Cam K, Simsek F, Yuksel M, Turkeri L, Haklar G, Yalcin S and Akdas A: The role of reactive oxygen species and apoptosis in the pathogenesis of varicocele in a rat model and efficiency of vitamin E treatment. Int J Androl 27: 228-233, 2004.

23. Mostafa T, Rashed L, Nabil N and Amin R: Seminal BAX and BCL2 gene and protein expressions in infertile men with varicocele. Urology 84: 590-595, 2014.

24. Alfadda AA and Sallam RM: Reactive oxygen species in health and disease. J Biomed Biotechnol 2012: 936486, 2012.

25. Bryan HK, Olayanju A, Goldring CE and Park BK: The Nrf2 cell defence pathway: Keap1-dependent and -independent mechanisms of regulation. Biochem Pharmacol 85: 705-717, 2013.

26. Nazima B, Manoharan V and Miltonprabu S: Oxidative stress induced by cadmium in the plasma, erythrocytes and lymphocytes of rats: Attenuation by grape seed proanthocyanidins. Hum Exp Toxicol 35: 428-447, 2016

27. Lin KN, Lin ML and Wei EQ: Protective effect of grape seed proanthocyanidin on cultured $\mathrm{RGC}-5$ cells against $\mathrm{CoCl}_{2}$-induced hypoxic injury. Zhejiang Da Xue Xue Bao Yi Xue Ban 44: 24-29, 2015 (In Chinese)
28. Khosravanian N, Razi M, Farokhi F and Khosravanian H: Testosterone and vitamin $\mathrm{E}$ administration up-regulated varicocele-reduced Hsp70-2 protein expression and ameliorated biochemical alterations. J Assist Reprod Genet 31: 341-354, 2014.

29. Lee JD, Lee TH, Cheng WH and Jeng SY: Involved intrinsic apoptotic pathway of testicular tissues in varicocele-induced rats. World J Urol 27: 527-532, 2009.

30. Barqawi A, Caruso A and Meacham RB: Experimental varicocele induces testicular germ cell apoptosis in the rat. J Urol 171: 501-503, 2004.

31. Zhang K, Wang Z, Wang H, Fu Q, Zhang H and Cao Q: Hypoxia-induced apoptosis and mechanism of epididymal dysfunction in rats with left-side varicocele. Andrologia 48: 318-324, 2016.

32. Du J and Dianjun G: Cell cycle specificity of spermatogenic cell apoptosis in rats with experimental varicocele. Clin Lab 59: 851-859, 2013.

33. Naughton CK, Nangia AK and Agarwal A: Pathophysiology of varicoceles in male infertility. Hum Reprod Update 7: 473-481, 2001.

34. Ricci JE, Muñoz-Pinedo C, Fitzgerald P, Bailly-Maitre B, Perkins GA, Yadava N, Scheffler IE, Ellisman MH and Green DR: Disruption of mitochondrial function during apoptosis is mediated by caspase cleavage of the p75 subunit of complex I of the electron transport chain. Cell 117: 773-786, 2004.

35. Sharma RK, Pasqualotto FF, Nelson DR, Thomas AJ Jr and Agarwal A: The reactive oxygen species-total antioxidant capacity score is a new measure of oxidative stress to predict male infertility. Hum Reprod 14: 2801-2807, 1999.

36. Sato M, Bagchi D, Tosaki A and Das DK: Grape seed proanthocyanidin reduces cardiomyocyte apoptosis by inhibiting ischemia/reperfusion-induced activation of JNK-1 and C-JUN. Free Radic Biol Med 31: 729-737, 2001

37. Song Q, Shi Z, Bi W, Liu R, Zhang C, Wang K and Dang X: Beneficial effect of grape seed proanthocyanidin extract in rabbits with steroid-induced osteonecrosis via protecting against oxidative stress and apoptosis. J Orthop Sci 20: 196-204, 2015.

38. Said TM, Paasch U, Glander HJ and Agarwal A: Role of caspases in male infertility. Hum Reprod Update 10: 39-51, 2004.

39. Palanisamy K, Krishnaswamy R, Paramasivan P, Chih-Yang H and Vishwanadha VP: Eicosapentaenoic acid prevents TCDD-induced oxidative stress and inflammatory response by modulating MAP kinases and redox-sensitive transcription factors. Br J Pharmacol 172: 4726-4740, 2015.

40. Jiang X, Bai Y, Zhang Z, Xin Y and Cai L: Protection by sulforaphane from type 1 diabetes-induced testicular apoptosis is associated with the up-regulation of Nrf2 expression and function. Toxicol Appl Pharmacol 279: 198-210, 2014.

41. Güçlü A, Yonguç N, Dodurga Y, Gündoğdu G, Güçlü Z, Yonguç T, Adıgüzel E and Turkmen K: The effects of grape seed on apoptosis-related gene expression and oxidative stress in streptozotocin-induced diabetic rats. Ren Fail 37: 192-197, 2015.

42. Akdemir S, Gurocak S, Konac E, Ure I, Onen HI, Gonul II, Sozen S and Menevse A: Different surgical techniques and L-carnitine supplementation in an experimental varicocele model. Andrologia 46: 910-916, 2014. 